\title{
AN INVESTIGATION ON THE POST-LOCAL-BUCKLING ANALYSIS OF I-SECTION STRUTS USING FINITE STRIP METHOD
}

\author{
H.R. Ovesy ${ }^{1, *}$, J. Loughlan ${ }^{2}$ and S.A.M. Ghannadpour ${ }^{3}$ \\ ${ }^{1}$ Aerospace Engineering Department and Centre of Excellence in Computational Aerospace Engineering, \\ Amirkabir University of Technology, Tehran, Iran \\ ${ }^{2}$ Department of Aeronautical and Automotive Engineering, Loughborough University, \\ Leicestershire LE11 3TU, UK \\ ${ }^{3}$ Space Engineering Dept., Faculty of New Technologies and Energy Engineering, Shahid Beheshti \\ University, G.C., Evin, 1983963113, Tehran, Iran \\ *(Corresponding author: E-mail: ovesy@aut.ac.ir)
}

Received: 29 August 2008; Revised: 22 November 2008; Accepted: 26 November 2008

\begin{abstract}
Two finite strip methods are developed for predicting the geometrically non-linear response of I-sections with simply supported ends when subjected to uniform end shortening in their plane. Although the formulations of both finite strip methods are based on the concept of the principle of minimum potential energy, the first finite strip method utilizes a semi-energy finite strip, whereas in the second finite strip method (which is designated by the name full-energy finite strip method) all the displacements are postulated by the appropriate shape functions. Both finite strip methods are then applied to analyze the post-local-buckling behavior of some representative I-section struts.
\end{abstract}

Keywords: Geometric Non-linear; Post-Local-Buckling; Semi-Energy; Finite Strip; I- Section

\section{INTRODUCTION}

Prismatic plates and plate structures are often employed in situations where they are subjected to in-plane compressive loading. Thus it is important to accurately predict the buckling and post buckling behavior of such structures. The post-local-buckling behavior of elastic plates or plate structures is a geometric non-linear problem. Among the energy-based approximate methods for the case of prismatic structures, the finite strip method (FSM) [1], which is a special form of the finite element method, has proved to be a capable tool for analyzing the post-buckling behavior of plates and plate structures. A typical example of a strut that is modeled by finite strips is shown in Figure 1.
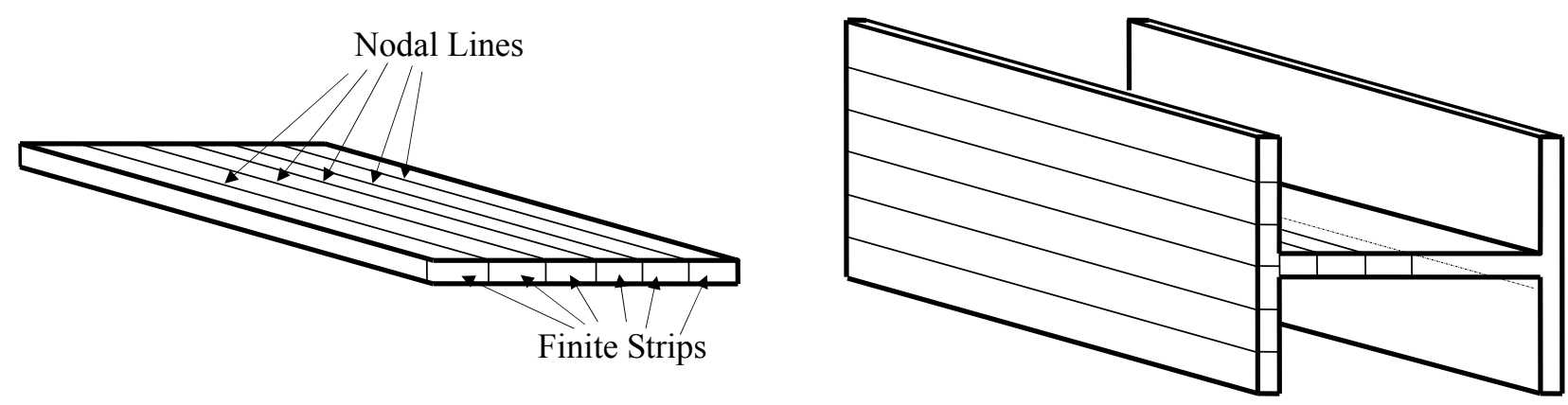

Figure 1. Plate Structures Discretized by Finite Strips 
Early works concerned with the use of the FSM in predicting the geometrically non-linear response of single rectangular plates and prismatic plate structures are those of Graves Smith and Sridharan [1, $2 \& 3]$ and Hancock [4]. These authors consider the post-buckling behaviour of plate structures subjected to uniform [ $1 \& 3]$ or linearly varying $[2,4]$ end shortening, with each component plate of the structure having simply supported ends. The elastic post-buckling response of channel section struts [1] and rectangular box columns [3] are investigated by Graves Smith and Sridharan. Hancock uses the finite strip method to investigate the post-buckling behaviour of square box and I-section columns. In the finite strip methods developed by the aforementioned authors, in-plane displacement fields are postulated in addition to the out-of-plane displacement field. Hence, these methods are to be designated as the full-energy FSM in the current paper. Since the time of the early publications discussed above, there has been a significant amount of work published on the application of the FSM to post-local-buckling behaviour of plates and plate structures. Dawe et al. [5] have developed a semi-analytical FSM for analyzing the post-local-buckling problem of composite plate structures. The finite strip properties are based on the use of both classical plate theory and first-order shear deformation plate theory, and the method is applied to analyze the post-local-buckling behaviour of box sections and top-hat-stiffened and blade-stiffened panels subjected to progressive uniform end shortening. Kwon and Hancock [6] have developed a non-linear elastic spline FSM to study the post-buckling behaviour of isotropic thin-walled sections undergoing local and distortional buckling. Azhari and Bradford [7] have developed a non-linear semi-analytical FSM for analyzing the elastic post-local-buckling behaviour of geometrically imperfect plate assemblies. The plate assemblies are assumed to be made of isotropic materials. In this method, the finite strip displacement functions, used earlier by Graves Smith and Sridharan [1], are augmented with so-called bubble functions, which are extra modes associated with internal or nodeless degrees of freedom. The method is deployed to study the post-local-buckling behaviour of channels under various compression eccentricities, and I-section members under pure compression and pure bending. An energy-based approximate method, referred to as the semi-energy method by Rhodes \& Harvey [8], was first used by Marguerre [9] in 1937, and has since been used by various researchers. More recently, Ovesy et al. [10-12] have developed a semi-energy post-local-buckling FSM in which the out-of-plane displacement of the finite strip is the only displacement which is postulated by a deflected form as opposed to that mentioned previously with respect to the full-energy FSM. The developed semi-energy FSM has been applied to analyze the post-local-buckling behavior of thin flat plates [10], open channel section [11] and box section struts [12]. Although a considerable amount of work has been published on the post-local-buckling behavior of plates and plate structures by using different versions of FSM, no work has been reported in which a thorough comparison is made between the semi-energy FSM and the more conventional full-energy FSMs, when applied to the post local- buckling analysis of I-sections. This task is fulfilled in this paper by developing a semi-energy post-local-buckling finite strip method as well as a full-energy finite strip method based on the theory proposed by Dawe et al. [5]. The developed finite strip methods are then applied to analyze the post-local-buckling behavior of some representative I-sections.

\section{THEORETICAL DEVELOPMENTS}

In this section, the fundamental elements of the theory for the developed finite strip methods are briefly outlined. It is noted that a perfectly flat finite strip made up of a linear isotropic material (with a constant modulus of elasticity $\mathrm{E}$ and Poisson ratio $v$ ) is assumed throughout the theoretical developments of this paper. The finite strip, which is schematically shown in Figure 2, is of thickness t. It may be noted that $u, v$ and $w$ correspond to the mid-plane displacements, and the finite strip is assumed to be simply supported out-of-plane at the loaded ends (i.e. at ends $x=0 \& \mathrm{~L}$ ). Moreover, the finite strip is assumed to be subjected to an in-plane compressive loading acting 
through frictionless rigid platens such that uniform end shortening $\bar{u}$ occurs at end $x=\mathrm{L}$ only (see Figure 2). The boundary conditions at loaded ends of the finite strip are summarized as follows:

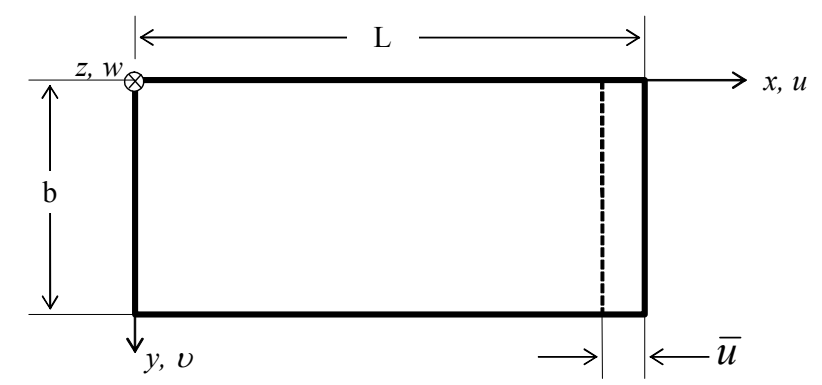

Figure 2. A Finite Strip Subjected to a Prescribed Uniform End Shortening

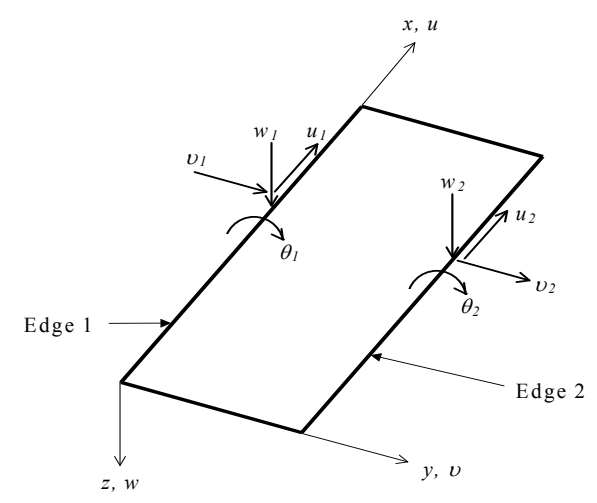

Figure 3. Displacement Parameters of a Finite Strip

$$
w=M_{x}=N_{x y}=0 \quad \text { at } \quad x=0 \& L \text { and } u= \begin{cases}0 & \text { at } \quad x=0 \\ -\bar{u} & \text { at } \quad x=L\end{cases}
$$

It is emphasized that the Classical Plate Theory (CPT) is applied in the remaining of the paper. As a result of this assumption, the Kirchhoff normalcy condition is incorporated, and thus:

$$
\begin{aligned}
& u_{t}(x, y, z)=u(x, y)-z \frac{\partial w(x, y)}{\partial x} \\
& v_{t}(x, y, z)=v(x, y)-z \frac{\partial w(x, y)}{\partial y} \\
& w_{t}(x, y, z)=w(x, y)
\end{aligned}
$$

where $u_{t}, v_{t}$ and $w_{t}$ are components of displacement at a general point, whilst $u, v$ and $w$ are similar components at the middle surfaces $(z=0)$.

On the assumption that the plate is in a state of plane stress, the stress-strain relationship at a general point for the plate becomes:

$$
\overline{\boldsymbol{\sigma}}=\left\{\begin{array}{l}
\bar{\sigma}_{x} \\
\bar{\sigma}_{y} \\
\bar{\tau}_{x y}
\end{array}\right\}=\frac{E}{1-v^{2}}\left[\begin{array}{ccc}
1 & v & 0 \\
v & 1 & 0 \\
0 & 0 & \frac{1-v}{2}
\end{array}\right] . \bar{\varepsilon} ; \bar{\varepsilon}=\left\{\begin{array}{l}
\bar{\varepsilon}_{x} \\
\bar{\varepsilon}_{y} \\
\bar{\gamma}_{x y}
\end{array}\right\}
$$

Where $\bar{\sigma}$ and $\overline{\boldsymbol{\varepsilon}}$, respectively, correspond to the stresses and strains at a general point. It is noted that these stresses and strains include the components corresponding to the membrane and bending contributions as outlined below :

$$
\bar{\sigma}=\sigma+\sigma_{b} ; \bar{\varepsilon}=\varepsilon+\varepsilon_{b}
$$


It is also noted that the relationship between $\sigma$ and $\varepsilon$, which correspond to the membrane contribution, is similar to that given by Eq. 3. The same relationship also applies between $\sigma_{b}$ and $\boldsymbol{\varepsilon}_{b}$, which correspond to the bending contribution. Moreover, the membrane strain $\varepsilon$ can be subdivided into its linear $\boldsymbol{\varepsilon}_{\boldsymbol{l}}$ and non-linear $\boldsymbol{\varepsilon}_{\boldsymbol{n}}$ components as given below:

$$
\boldsymbol{\varepsilon}=\boldsymbol{\varepsilon}_{l}+\boldsymbol{\varepsilon}_{n l}=\left\{\begin{array}{c}
\frac{\partial u}{\partial x} \\
\frac{\partial v}{\partial y} \\
\frac{\partial u}{\partial y}+\frac{\partial v}{\partial x}
\end{array}\right\}+\left\{\begin{array}{c}
\frac{1}{2}\left(\frac{\partial w}{\partial x}\right)^{2} \\
\frac{1}{2}\left(\frac{\partial w}{\partial y}\right)^{2} \\
\frac{\partial w}{\partial x} \frac{\partial w}{\partial y}
\end{array}\right\}
$$

It is also noted that within the context of CPT the bending strains $\boldsymbol{\varepsilon}_{b}$ are expressed by the following equations:

$$
\boldsymbol{\varepsilon}_{\boldsymbol{b}}=\left\{\begin{array}{c}
-z \frac{\partial^{2} w}{\partial x^{2}} \\
-z \frac{\partial^{2} w}{\partial y^{2}} \\
-2 z \frac{\partial^{2} w}{\partial x \partial y}
\end{array}\right\}
$$

Since the potential energy of external loads is zero for the strip under consideration, the total potential energy of the strip $V_{s}$ is simply equal to the its strain energy $U_{s}$ (i.e. $V_{s}=U_{s}$ ) which is given below:

$$
U_{s}=\frac{1}{2} \iiint\left(\bar{\sigma}_{x} \bar{\varepsilon}_{x}+\bar{\sigma}_{y} \bar{\varepsilon}_{y}+\bar{\tau}_{x y} \bar{\gamma}_{x y}\right) d x d y d z
$$

By substituting $\bar{\sigma}$ and $\bar{\varepsilon}$ from Eq. 4 into Eq. 7 and rearranging, the total strain energy of the strip $U_{s}$ can be expressed by the following Equations:

$$
U_{s}=U_{m s}+U_{b s}
$$

Where $U_{m s}$ designates the membrane strain energy of the strip and is given by:

$$
U_{m s}=\frac{1}{2} \iiint\left(\sigma_{x} \varepsilon_{x}+\sigma_{y} \varepsilon_{y}+\tau_{x y} \gamma_{x y}\right) d x d y d z
$$

and $U_{b s}$ designates the bending strain energy of the strip and is given by: 


$$
U_{b s}=\frac{1}{2} \iiint\left(\sigma_{b x} \varepsilon_{b x}+\sigma_{b y} \varepsilon_{b y}+\tau_{b x y} \gamma_{b x y}\right) d x d y d z
$$

The membrane strain energy $U_{m s}$ is further expanded by substituting for $\boldsymbol{\sigma}$ in terms of $\varepsilon$ using Eq. 3 , and subsequently carrying out the integration in the $z$ direction. This gives:

$$
U_{m s}=\frac{\mathrm{A}}{2} \iint\left(\varepsilon_{x}^{2}+\varepsilon_{y}^{2}+2 v \varepsilon_{x} \varepsilon_{y}+\frac{1-v}{2} \gamma_{x y}^{2}\right) d x d y
$$

Where $\mathrm{A}=\mathrm{Et}_{\mathrm{s}} /\left(1-v^{2}\right)$ is the so-called in-plane stiffness of the strip.

The bending strain energy $U_{b s}$ is also expanded by using Eq. 3, and subsequently substituting for $\boldsymbol{\varepsilon}_{b}$ by implementing Eq. 6, and finally carrying out the integration in the $z$ direction. This gives:

$$
U_{b s}=\frac{D^{2}}{2} \int_{0}^{b_{s}} \int_{0}^{L}\left\{\left(\frac{\partial^{2} w}{\partial x^{2}}+\frac{\partial^{2} w}{\partial y^{2}}\right)^{2}+2(1-v)\left[\left(\frac{\partial^{2} w}{\partial x \partial y}\right)^{2}-\frac{\partial^{2} w}{\partial x^{2}} \frac{\partial^{2} w}{\partial y^{2}}\right]\right\} d x d y
$$

Where $\mathrm{D}=\mathrm{Et}^{3} / 12\left(1-v^{2}\right)$ is the so-called bending stiffness of the strip.

\subsection{Theoretical Developments of Semi-energy Finite Strip}

A more detailed coverage of the theoretical aspects and developments of the semi-energy finite strip approach are given in an earlier publication by Ovesy et al [10], which pertains to the compressive post-buckling behaviour of thin plates, and the reader is referred to this reference for a deeper insight into the method. In the present paper, however, the authors feel that in the application of the approach to structural sections a theoretical presentation of the method is, indeed, necessary but that this should only include the dominating features of the method and thus these are detailed in the paper in a reduced outline presentation of the approach.

As mentioned earlier, in the semi-energy method, the out-of-plane displacement of the finite strip is the only displacement which is postulated by a deflected form. Then, the postulated deflected form is substituted into von Kármán's compatibility equation which is solved exactly to obtain the form of the in-plane displacements that correspond to the postulated form of the out-of-plane displacement. The von Kármán's compatibility equation for large deflections of plate is given by Eq. 13.

$$
\nabla^{4} \mathrm{~F}=\frac{\partial^{4} \mathrm{~F}}{\partial x^{4}}+2 \frac{\partial^{4} \mathrm{~F}}{\partial x^{2} \partial y^{2}}+\frac{\partial^{4} \mathrm{~F}}{\partial y^{4}}=\mathrm{E}\left\{\left(\frac{\partial^{2} w}{\partial x \partial y}\right)^{2}-\left(\frac{\partial^{2} w}{\partial x^{2}}\right)\left(\frac{\partial^{2} w}{\partial y^{2}}\right)\right\}
$$

In this equation the function $\mathrm{F}$ (i.e. $\mathrm{F}=\mathrm{F}(x, y)$ ) which is known as the Airy stress function is defined as follows: 


$$
\begin{aligned}
& \sigma_{x}=\frac{\partial^{2} \mathrm{~F}(x, y)}{\partial y^{2}} \\
& \sigma_{y}=\frac{\partial^{2} \mathrm{~F}(x, y)}{\partial x^{2}} \\
& \tau_{x y}=-\frac{\partial^{2} \mathrm{~F}(x, y)}{\partial x \partial y}
\end{aligned}
$$

It is noted that the function given by Eq. 15 has been found to be suitable for the representation of the out-of-plane displacement field for the developed semi-energy finite strip formulation [Ovesy et.al [10]]. It is emphasized that the developed semi-energy finite strip is based on a single term representation of the out-of-plane displacement.

$w=\mathrm{f}_{w} \sin \left(\frac{\mathrm{M} x}{2}\right)$

where

$$
\mathrm{f}_{w}=\left(1-3 \eta^{2}+2 \eta^{3}\right) w_{1}+b\left(\eta-2 \eta^{2}+\eta^{3}\right) \theta_{1}+\left(3 \eta^{2}-2 \eta^{3}\right) w_{2}+b\left(\eta^{3}-\eta^{2}\right) \theta_{2}
$$

and $\mathrm{M}=2 n \pi / L, \eta=y / b$ and $w_{1}, \theta_{1}, w_{2}, \theta_{2}$ are the undetermined out-of-plane nodal displacement parameters along edges 1 and 2 of the strip (see Figure 3). These nodal displacement parameters are also called the degrees of freedom (DOF). It is noted that $n$ represents the number of buckle half waves along the length of the strip. The out-of-plane displacement $w$ is then substituted into von Kármán's compatibility equation in order to find the corresponding in-plane displacement functions. In this process, the stress function $\mathrm{F}$ may be considered in two parts; one part varying periodically with $x$, and the other part constant with respect to $x$, i.e.

$$
\mathrm{F}=\mathrm{F}_{1}+\mathrm{F}_{2} \cos (\mathrm{M} x)
$$

Substituting F from Eq. 16 and $w$ from Eq. 15 in Eq. 13 and imposing the boundary conditions as Eq. 1, the in-plane $u$ displacement function corresponding to the assumed out-of-plane $w$ displacement can eventually be derived as Eq. 17.

$$
u=-\frac{\bar{u}}{L} x+\mathrm{f}_{u}(y) \sin (\mathrm{M} x)
$$

where

$$
\mathrm{f}_{u}(y)=\mathrm{f}_{u}=\frac{\mathrm{M}}{8}\left(\Psi^{\prime \prime}+v \mathrm{M}^{2} \Psi-\frac{\mathrm{f}_{w}^{2}}{2}\right)
$$

and $\Psi$ can be found from the following equation:

$$
\Psi^{\prime \prime \prime \prime}-2 \mathrm{M}^{2} \Psi^{\prime \prime}+\mathrm{M}^{4} \Psi=\left(\mathrm{f}_{w}^{\prime}\right)^{2}-\left(\mathrm{f}_{w} \mathrm{f}_{w}^{\prime \prime}\right)
$$

It is noted that the prime sign designates the derivatives of the corresponding parameter with respect to $\mathrm{y}$, thus, for example $\mathrm{f}_{w}^{\prime \prime}=\partial^{2} \mathrm{f}_{w} / \partial y^{2}$. It is also noted that the first term on the right hand 
side of Eq. 17 represents the prescribed uniform end shortening. The amplitude of the second term evaluated at $y=0$ and $y=b$ (i.e. $\left.\mathrm{f}_{u}\right|_{y=0}$ and $\left.\mathrm{f}_{u}\right|_{y=\mathrm{b}}$ ) represents the local degrees of freedom $u_{1}$ and $u_{2}$ respectively (see Figure 3). The solution of Eq. 19 is composed of two parts; the particular integral solution, and the complementary function solution. The particular integral solution depends on the function $\mathrm{f}_{w}$ only, thus it can be conveniently evaluated. The complementary function solution is as follows:

$$
\Psi_{\text {C.F. }}=\mathrm{B}_{1} \sinh (\mathrm{M} y)+\mathrm{B}_{2} \cosh (\mathrm{M} y)+\mathrm{B}_{3} y \sinh (\mathrm{M} y)+\mathrm{B}_{4} y \cosh (\mathrm{M} y)
$$

The coefficients $\mathrm{B}_{1} \rightarrow \mathrm{B}_{4}$ are unknown at present, but it is assumed that these coefficients and subsequently $\Psi_{\mathrm{C} . \mathrm{F}}$ and $\Psi$ are known so that the analysis to find the in-plane displacements can be completed. After substituting for the appropriate terms and simplification, the degrees of freedom $u_{1}$ and $u_{2}$ can be found explicitly in terms of $w_{1}, \theta_{1}, w_{2}, \theta_{2}, \mathrm{~b}, \mathrm{M}, v$ and $\mathrm{B}_{1} \rightarrow \mathrm{B}_{4}$. It is shown by Ovesy et al [10] that the in-plane displacement $v$ function can be derived as follow:

$v=v \frac{\bar{u}}{L} y+\mathrm{I}_{1 v}+\mathrm{f}_{v} \cos (\mathrm{M} x)-\left.\mathrm{f}_{v}\right|_{y=0}+\left.v\right|_{x=0, y=0}$

where

$$
\mathrm{f}_{\mathrm{v}}(y)=\mathrm{f}_{\mathrm{v}}=\frac{1}{8}\left[\Psi^{\prime \prime \prime}-(2+v) \mathrm{M}^{2} \Psi^{\prime}+\mathrm{f}_{w} \mathrm{f}_{w}^{\prime}\right]
$$

and

$$
\mathrm{I}_{1 \mathrm{v}}=-\int_{0}^{y}\left[\frac{v \mathrm{M}^{2}}{16} \mathrm{f}_{w}^{2}+\frac{\left(\mathrm{f}_{w}^{\prime}\right)^{2}}{4}\right] d y
$$

The above equation describes the in-plane $v$ displacement function corresponding to the assumed out-of-plane $w$ displacement function. The first term on the right hand side of Eq. 21 describes the transverse in-plane expansion of the strip, which occurs due to the Poisson's ratio effect. The second term (i.e. $\mathrm{I}_{1 \mathrm{v}}$ ) describes the transverse in-plane movement of the longitudinal fibers of the strip. This movement, which is constant along the length of a given fiber, varies from a minimum value of zero at edge $y=0$ to its maximum value at the edge $y=b$. The third term describes the in-plane waviness of the longitudinal fibers. The amplitude of this term evaluated at $y=0$ and $y=b$ (i.e. $\left.\mathrm{f}_{v}\right|_{y=0}$ and $\left.\mathrm{f}_{v}\right|_{y=b}$ ) represents the local degrees of freedom $v_{1}$ and $v_{2}$ respectively (see Figure 3). These degrees of freedom can explicitly be described in terms of $w_{1}, \theta_{1}, w_{2}, \theta_{2}, \mathrm{~b}, \mathrm{M}, v$ and $\mathrm{B}_{1}-\mathrm{B}_{4}$. Finally, the fourth term (which is equivalent to $v_{1}$ ) and the fifth term on the right hand side of Eq. 21 represent values which remain constant at all points on a given strip. The existence of the fourth term on the right hand side of Eq. 21 (i.e. $-\left.f_{v}\right|_{y=0}=-v_{1}$ ) allows the point $(x=0, y=0)$ to be treated as a reference point in terms of its deflection being connected to another strip or being restrained. It is noted that the function $\Psi$ is not yet fully known due to the coefficients $\mathrm{B}_{1} \rightarrow \mathrm{B}_{4}$ being unknown. These coefficients are obtained by treating them as unknowns, and solving for them while all other parameters, including all degrees of freedom (i.e. $u_{1}, v_{1}, w_{1}, \theta_{1}$, $u_{2}, v_{2}, w_{2}$ and $\theta_{2}$ ), are assumed to be known. Thus, the coefficients $\mathrm{B}_{1} \rightarrow \mathrm{B}_{4}$ are explicitly described in terms of $u_{1}, v_{1}, w_{1}, \theta_{1}, u_{2}, v_{2}, w_{2}, \theta_{2}, \mathrm{~b}, \mathrm{M}$ and $v$. Having found the coefficients $\mathrm{B}_{1} \rightarrow \mathrm{B}_{4}$, they are substituted in Eq. 20 to find the complementary function solution. Subsequently, the combination of the complementary function and particular integral solutions are used to substitute for $\Psi$ in the expressions describing the in-plane displacement $u$ (i.e. Eqs. 17-19) and $v$ (i.e. Eqs. 21-23). 
Having obtained the form of the in-plane displacements that correspond to the postulated form of the out-of-plane displacement, the membrane strain energy of the strip is now evaluated by using Eq. 11, and substituting for $\varepsilon$ in terms of the derivatives of the in-plane and out-of-plane displacements employing Eqs. 5, 15, 17 and 21. After performing the integration with respect to $x$ between 0 and $\mathrm{L}$, the following expression is obtained:

$$
\begin{aligned}
\mathrm{U}_{\mathrm{ms}}= & \frac{\mathrm{ELt}}{2} \int_{0}^{\mathrm{b}}\left\{\left(\frac{\overline{\mathrm{u}}}{\mathrm{L}}\right)^{2}+\frac{\mathrm{M}^{4}}{128}\left(\Psi^{\prime \prime}\right)^{2}+\frac{\mathrm{M}^{6}}{64} v \Psi \Psi^{\prime \prime}+\frac{\mathrm{M}^{8}}{128} \Psi^{2}+\right. \\
& \left.\frac{\mathrm{M}^{6}}{64}(1+v)\left(\Psi^{\prime}\right)^{2}+\frac{\mathrm{M}^{4}}{256} \mathrm{f}_{w}^{4}-\frac{\mathrm{M}^{2} \overline{\mathrm{u}}}{8 \mathrm{~L}} \mathrm{f}_{w}^{2}\right\} d y
\end{aligned}
$$

The bending strain energy $U_{b s}$ is expanded by substituting for $w$ from Eq. 15 into Eq. 12 . Then, the total potential energy of the strip can be found by adding the membrane strain energy of the strip to the bending strain energy (see Eq. 8). The total potential energy for a strip is re-arranged in the form given below:

$$
V_{s}=U_{s}=\frac{1}{2} \mathrm{~d}_{\mathrm{s}}^{\mathrm{T}}\left(\mathrm{K}-\frac{\bar{u}}{l} \mathrm{~K}^{*}\right) \mathrm{d}_{\mathrm{s}}+\frac{1}{6} \mathrm{~d}_{\mathrm{s}}^{\mathrm{T}} \mathrm{K}_{1} \mathrm{~d}_{\mathrm{s}}+\frac{1}{12} \mathrm{~d}_{\mathrm{s}}^{\mathrm{T}} \mathrm{K}_{2} \mathrm{~d}_{\mathrm{s}}
$$

Here $\mathrm{K}, \mathrm{K}^{*}, \mathrm{~K}_{2}$ and $\mathrm{K}_{1}$ are symmetric square stiffness matrices. The coefficients of $\mathrm{K}$ and $\mathrm{K}^{*}$ are constant whilst those of $\mathrm{K}_{1}$ and $\mathrm{K}_{2}$ are linear and quadratic functions, respectively, of the displacements. The column matrix $\mathrm{d}_{\mathrm{s}}$ contains the strip degrees of freedom. In evaluating $V_{s}$, all the integrations in the $x$ and $y$ directions are determined analytically.

For the whole structure, comprising an assembly of finite strips, the total potential energy is simply the summation of the potential energies of the individual finite strips. Correspondingly, whole structure matrices which are equivalent of those appearing in Eq. 25 for the individual finite strip are generated by appropriate summations in the standard fashion. Thus, the potential energy for the whole structure can be expressed as:

$$
\bar{V}=\frac{1}{2} \overline{\mathrm{d}}^{\mathrm{T}}\left(\overline{\mathrm{K}}-\frac{\bar{u}}{l} \overline{\mathrm{K}}^{*}\right) \overline{\mathrm{d}}+\frac{1}{6} \overline{\mathrm{d}}^{\mathrm{T}} \overline{\mathrm{K}}_{1} \overline{\mathrm{d}}+\frac{1}{12} \overline{\mathrm{d}}^{\mathrm{T}} \overline{\mathrm{K}}_{2} \overline{\mathrm{d}}
$$

where the overbar indicates a whole-structure quantity.

The structure equilibrium equations are obtained by applying the principle of minimum potential energy. The partial differentiation of the structure potential energy with respect to each degree of freedom in turn gives a set of non-linear equilibrium equations as outlined below:

$$
\left(\overline{\mathrm{K}}-\frac{\bar{u}}{l} \overline{\mathrm{K}}^{*}+\frac{1}{2} \overline{\mathrm{K}}_{1}+\frac{1}{3} \overline{\mathrm{K}}_{2}\right) \overline{\mathrm{d}}=0 \quad \text { or } \quad \overline{\mathrm{K}}_{\mathrm{s}} \cdot \mathrm{d}=0
$$

where $\overline{\mathbf{K}}_{\mathrm{S}}$ is the global/structural stiffness matrix, and $\overline{\mathrm{d}}$ is a vector, which includes the degrees of freedom for the whole structure. The latter set of equations needs to be modified by applying the appropriate boundary conditions. These equations can be solved using an iterative procedure and here it is the Newton-Raphson procedure that is used, in the manner described below. 
It is assumed that $\overline{\mathrm{d}}_{\mathrm{i}}$ is an approximate trial solution at a particular prescribed end shortening $\bar{u}$. In this case, $F\left(\overline{\mathrm{d}}_{\mathrm{i}}\right)$, which is defined by the following equation, denotes the error in the approximation:

$$
\mathrm{F}\left(\overline{\mathrm{d}}_{\mathrm{i}}\right)=\overline{\mathrm{K}}_{\mathrm{s}} \cdot \overline{\mathrm{d}}_{\mathrm{i}} \neq 0
$$

Applying the Newton-Raphson algorithm to this trial solution, an improved solution $\overline{\mathrm{d}}_{\mathrm{i}+1}$ is obtained by equating to zero the curtailed Taylor's expansion of $F\left(\bar{d}_{i+1}\right)$ in the neighborhood of $\bar{d}_{i}$, i.e.

$$
\mathrm{F}\left(\overline{\mathrm{d}}_{\mathrm{i}+1}\right) \cong \mathrm{F}\left(\overline{\mathrm{d}}_{\mathrm{i}}\right)+\frac{\partial \mathrm{F}\left(\overline{\mathrm{d}}_{\mathrm{i}}\right)}{\partial \overline{\mathrm{d}}_{\mathrm{i}}}\left(\overline{\mathrm{d}}_{\mathrm{i}+1}-\overline{\mathrm{d}}_{\mathrm{i}}\right)=0
$$

Differentiating Eq. 28 with respect to $\overline{\mathrm{d}}_{\mathrm{i}}$ gives

$$
\frac{\partial \mathrm{F}\left(\overline{\mathrm{d}}_{\mathrm{i}}\right)}{\partial \overline{\mathrm{d}}_{\mathrm{i}}}=\overline{\mathrm{K}}-\frac{\bar{u}}{l} \overline{\mathrm{K}}^{*}+\overline{\mathrm{K}}_{1}+\overline{\mathrm{K}}_{2}=\overline{\mathrm{K}}_{\mathrm{T}}
$$

where $\overline{\mathrm{K}}_{\mathrm{T}}$ is the symmetric tangent stiffness matrix evaluated at $\overline{\mathrm{d}}_{\mathrm{i}}$. Eq. 29 can now be expressed as

$$
\overline{\mathrm{K}}_{\mathrm{T}} \Delta \overline{\mathrm{d}}_{\mathrm{i}}=-\mathrm{F}\left(\overline{\mathrm{d}}_{\mathrm{i}}\right)
$$

where $\Delta \overline{\mathrm{d}}_{\mathrm{i}}$ is the correction at the $i$ th iteration. Having obtained the solution of this equation for $\Delta \overline{\mathrm{d}}_{\mathrm{i}}$, the improved solution at the end of the $i$ th iteration for a prescribed value of $\bar{u}$, is then given by

$$
\overline{\mathrm{d}}_{\mathrm{i}+1}=\overline{\mathrm{d}}_{\mathrm{i}}+\Delta \overline{\mathrm{d}}_{\mathrm{i}}
$$

In the present study, the iterative procedure is repeated until the following convergence criteria are satisfied:

$$
\sqrt{\frac{\sum \Delta \overline{\mathrm{d}}_{\mathrm{i}}^{2}}{\sum \overline{\mathrm{d}}_{\mathrm{i}+1}{ }^{2}}}<10^{-7}
$$

and

$$
\sqrt{\sum \mathrm{F}_{i}^{2}}<10^{-5}
$$

where the summation relates to all active degrees of freedom, and $F_{i}=F\left(\bar{d}_{i}\right)$.

Once the global equilibrium equations are solved and the nodal degrees of freedom are found for a particular prescribed end shortening, it is possible to calculate the out-of-plane displacement $w$ in any finite strip using Eq. 15 directly. The calculation of the in-plane displacements $u$ and $v$ is achieved after finding $\mathrm{I}_{10}, \Psi$ and the derivatives of $\Psi$.

The stresses and strains at any point in any strip are calculated using Eqs. 3 to 6 . 
The longitudinal force/load acting on a strip at a given cross-section along the strip length $\mathrm{P}_{\mathrm{s}}$ is determined by integrating the longitudinal mid-plane stresses $\sigma_{x}$ over the strip cross-sectional area, i.e.

$$
\mathrm{P}_{s}=-\int_{0}^{l} \int_{0}^{\mathrm{b}} \sigma_{x} d x d y
$$

The total longitudinal force/load acting on a plate or plate structures at a given cross section along their length, corresponding to a prescribed end shortening, is obtained by summation of all strip forces $\mathrm{P}_{\mathrm{s}}$ (given by Eq. 35 ) at the same cross section, i.e.

$$
\mathrm{P}=\sum \mathrm{P}_{\mathrm{s}}
$$

It is emphasized that the positive $\mathrm{P}$ values represent compressional loads.

\subsection{Theoretical Developments of Full-energy Finite Strip}

Unlike the semi-energy method, which has been developed based on a single term concept only, the full-energy finite strip method employs a multi-term concept. The main difference between the two methods, however, lies in the fact that in the full-energy method, in addition to postulating a deflected form for the out-of-plane displacement, the in-plane displacements of the finite strip are also postulated by the appropriate deflected forms from the commencement of analysis. As far as the expression of the in-plane displacements and out-of-plane displacement in the full-energy method are concerned, in line with the work of Dawe et al. [5], the following forms are assumed:

$$
\begin{aligned}
& u=-\frac{\bar{u}}{L} x+\sum_{n=1}^{r u} \mathbf{f}_{n}^{\mathrm{u}} \sin \left(\frac{n \pi x}{L}\right) \\
& v=v \frac{\bar{u}}{L} y+\sum_{n=0}^{r v} \mathrm{f}_{n}^{\mathrm{v}} \cos \left(\frac{n \pi x}{L}\right) \\
& w=\sum_{n=1}^{r w} \mathrm{f}_{\mathrm{w}_{n}} \sin \left(\frac{n \pi x}{L}\right)
\end{aligned}
$$

where the $\mathrm{f}_{n}^{\mathrm{u}}, \mathrm{f}_{n}^{\mathrm{v}}$ and $\mathrm{f}_{\mathrm{w} n}$ are the appropriate transverse polynomial interpolation functions, involving undetermined nodal parameters. These functions are expressed below:

$$
\begin{aligned}
& \mathrm{f}_{n}^{u}(y)=\frac{1}{2}(1-2 \eta) u_{1 n}+\frac{1}{2}(1+2 \eta) u_{2 n} \\
& \mathrm{f}_{n}^{\mathrm{v}}(y)=\frac{1}{2}(1-2 \eta) v_{1 n}+\frac{1}{2}(1+2 \eta) v_{2 n} \\
& \mathrm{f}_{w n}=\left(1-3 \eta^{2}+2 \eta^{3}\right) w_{1 n}+b\left(\eta-2 \eta^{2}+\eta^{3}\right) \theta_{1 n}+\left(3 \eta^{2}-2 \eta^{3}\right) w_{2 n}+b\left(\eta^{3}-\eta^{2}\right) \theta_{2 n}
\end{aligned}
$$


where $\eta=y / b$ and the $u_{1 n}, u_{2 n}, v_{1 n}, v_{2 n}, w_{1 n}, \theta_{1 n}, w_{2 n}, \theta_{2 n}$ represent the degrees of freedom corresponding to the $n$th series' term along the length of the strip. It is seen that for a given term, the shape function $f_{\mathrm{w} n}$ is the same as that already used in connection with the semi-energy method (i.e. Eq. 15-b).

With the establishment of the finite strip displacement fields according to the above-mentioned equations, the rest of the analysis is carried out in the same manner as that already described with respect to semi-energy finite strip method. It is worth mentioning that in evaluating the potential energy of the full-energy strip, similar to the process followed for the semi-energy finite strip, all integrations in the $x$ and $y$ directions are determined analytically. Further details regarding theoretical developments of the full-energy finite strip approach are given in an earlier publication by Ovesy and Ghannadpour [13].

\section{RESULTS AND DISCUSSIONS}

The type of plate structure investigated is plain I-section struts whose cross-sectional geometry is depicted in Figure 1. It is noted that the present study is concerned exclusively with local buckling. Considering the fact that the transverse in-plane displacement $v$ and the out-of-plane displacement $\mathrm{w}$ are characterized by differing trigonometric series, it is further assumed that the in-plane and out-of-plane deflections at junctions are uncoupled. The plate components are assumed to be free to wave across their width along the junctions where the out-of-plane deflection of each of the plates vanishes separately i.e. $\sigma_{y}=0, w=0$ along the junctions. This assumption is a valid approximation for an initial post-local-buckling analysis. It is noted that it is neither possible nor necessary to define a global co-ordinate system for the plate structure as a whole due to the uncoupling between $v$ and $w$ displacements at junctions. Hence, within any component plate the local degrees of freedom in the interior can be used directly to relate to the structure. Since the local buckling mode of I-section strut is symmetric with respect to the axis passing through web centre parallel to the flanges, it was sufficient to analyze one half of the cross-section while the necessary symmetric boundary conditions were applied along the axis of symmetry. In order to reflect the symmetry of the problem in the longitudinal direction, the appropriate series' terms were chosen and used in the full-energy finite strip approach. That is to say, for $u$ the first three even terms $(n=$ $2,4,6)$ and for $v$ the first four even terms $(n=0,2,4,6)$ and for $w$ the first two odd terms $(n=1,3)$ were used. It is noted that in the semi-energy finite strip approach, only one term $(n=1)$ was used for $w$.

The number of strips $N_{s}$ which is needed for convergence is examined. For example, in the full energy method and for an I-section strut with a uniform thickness across the section and the strut length equal to the natural half-wave length with $b_{f} / b_{w}=0.4$, the post-buckling compressional stiffness ratio $\mathrm{S}^{*} / \mathrm{S}$ is equal to $0.6333,0.6319$ and 0.6314 for the number of strips $N_{S}$ equal to 24,36 and 48 respectively. Thus in the full energy method, the strips allocation corresponding to 16 strips to the half-web and 32 strips to the flange, resulting in a total of 48 strips for half the section is considered adequate. The same study resulted in a total of 24 strips in the semi-energy approach by assigning 8 identical strips to each of the plate elements meeting at the junction. It is worth mentioning that in presenting the results, the Poisson's ratio $v$ is assumed to be 0.3 and a common Young's modulus with the value within the range corresponding to steel module (i.e. $E=207000$ $\mathrm{MPa}$ ) was assumed for I-section. Figures 4 and 5 depict the initial post-buckling ( $\mathrm{S}^{*}$ ) to pre-buckling (S) compressional stiffness ratio (i.e. $\mathrm{S}^{*} / \mathrm{S}$ at the instant of local instability) for a number of I-section struts with a variety of geometric configurations. It is noted that the 
post-buckling compressional stiffness of the strut is defined by $\mathrm{S}^{*}=\partial \mathrm{P} / \partial \overline{\mathrm{u}}$, where $\mathrm{P}$ is the total longitudinal load of the struts. It is also noted that the pre-buckling compressional stiffness (i.e. $\mathrm{S}=\partial \mathrm{P} / \partial \overline{\mathrm{u}})$ is a constant value. In Figure 4, the strut length is equal to the natural half-wave lengths, whereas in Figure 5, the strut length is constant and equal to the web width. Moreover, In Figure 4, a uniform thickness across the section is assumed, whereas in Figure 5 three different flange-web thickness ratios are considered. It is worth mentioning that in Figure 4, the finite strip analyses were carried out only for those configurations that are marked with symbols on the graph. The smoothest curve was then passed through the points to show the general behaviour of the structures.

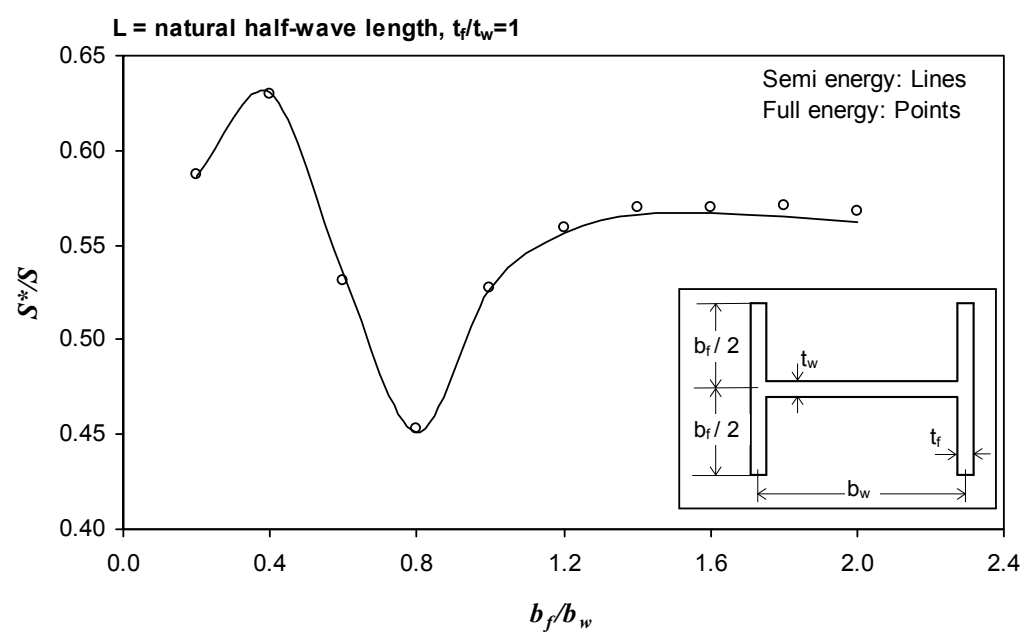

Figure 4. Post-Buckling Compressional Stiffness Ratio of an I-Section Strut at the Instant of Buckling

In Figure 5, however, over 200 different combinations of shape factors were analysed. Both figures indicate that for each curve, there is a particular shape factor which produces a minimum $\mathrm{S}^{*} / \mathrm{S}$ ratio. Prior to this shape factor, buckling is initiated in the web which is being restrained by the flanges against further deflection. Thereafter, the flanges start the buckling and the web restrains them. It is seen in Figure 5 that prior to the shape factor corresponding to the minimum $\mathrm{S}^{*} / \mathrm{S}$ ratio, where buckling is initiated in the web, the lower the $t_{f} / t_{w}$ ratio, the lesser the post-buckling stiffness. However, thereafter, where flange predominates buckling, the lower the $t_{f} / t_{w}$ ratio, the higher the $\mathrm{S}^{*} / \mathrm{S}$ values. This behaviour may be attributed to the fact that for web buckling, the thick flange provides a high degree of restraint on the thin web prior to and after the local buckling, causing an increase in the post-buckling compressional stiffness. However, when buckling is dominant in the flange, the thin web is incapable of imposing such restraints and consequently a substantial reduction in the stiffness is experienced. It is seen in both figures that the general agreement between the results obtained by the semi-energy approach and those obtained by the full-energy method are very good. It is also seen that when the flange gets thinner, i.e. the cases with $\mathrm{t}_{\mathrm{f}} / \mathrm{t}_{\mathrm{w}}=0.5$ in Figure 5 which are also tabulated in Table 1, and when the flange gets longer, i.e. the cases with higher $b_{f} / b_{w}$ ratios in Figure 4 which correspond to longer natural half-wave length, the increase in $b_{f} / b_{w}$ ratio has caused the semi-energy results to be more accurate by predicting lower values for post-buckling compressional stiffness compared to those achieved by full-energy method. This difference may be attributed to the fact that for the aforementioned cases, where the buckling of the section is highly dominated by that of the flange which is effectively free on both sides, the formulation of the semi-energy method in which the in-plane displacements are obtained by solving the von Kármán's compatibility equation is more accurate. Figure 6 represents 
the non-dimensional load-end shortening variation for struts with flange-web width ratio equal to 0.5. The length of the struts is assumed to be equal to their web width. It is noted that three different $\mathrm{t}_{\mathrm{f}} / \mathrm{t}_{\mathrm{w}}$ ratios are studied. The non-linear nature of the behaviour, which happens due to the effect of change in the buckle shape, can clearly be seen for all cases. Once again, a very good agreement is seen to exist between the results obtained by the semi-energy approach and those obtained by the full-energy method. It is to be noted that in the current semi-energy approach only one term is utilized, whereas several terms are implemented in the formulation of the full-energy method. Therefore, it is expected that the accuracy of the semi-energy approach will improve if more than one term is utilized in its formulation.

Table 1. Comparison of the Semi Energy Results $\left(S^{*} / S\right)$ with the Results of Full Energy

\begin{tabular}{c|c|c|c}
\hline \multirow{2}{*}{$\mathbf{b}_{\mathrm{f}} / \mathbf{b}_{\mathbf{w}}$} & \multicolumn{2}{|c|}{$\mathbf{S}^{*} / \mathbf{S}$} & \multirow{2}{*}{ Error (\%) } \\
\cline { 2 - 3 } & Semi energy & Full energy & \\
\hline 2.0 & 0.6615 & 0.6694 & 0.79 \\
1.8 & 0.6766 & 0.6826 & 0.60 \\
1.6 & 0.6930 & 0.6971 & 0.41 \\
1.4 & 0.7105 & 0.7127 & 0.22 \\
1.2 & 0.7284 & 0.7289 & 0.05 \\
1.0 & 0.7433 & 0.7443 & 0.10 \\
0.8 & 0.7356 & 0.7390 & 0.34 \\
0.7 & 0.6855 & 0.6872 & 0.17 \\
0.6 & 0.5223 & 0.5257 & 0.34 \\
0.5 & 0.4277 & 0.4329 & 0.52 \\
0.4 & 0.4928 & 0.4960 & 0.32 \\
0.2 & 0.5027 & 0.5052 & 0.25 \\
\hline
\end{tabular}

Figure 7 depicts the longitudinal mid-plane stress distributions across the cross-section of the struts with $t_{f} / t_{w}$ ratio equal to 1 , which is investigated earlier in connection with the load-end shortening variation with reference to Figure 6 . The stress variation is presented at the crest of the buckle at the load levels equal to twice and three times the buckling load. Once again due to the symmetry, the stress distributions are shown for only a half of the cross-section. A good agreement is seen to exist among the results obtained by both finite strip methods.

As expected, the maximum membrane stress occurs at the plate junction at the crest of the buckle for any specified level of loading. The figure show that, at the load levels under consideration, the flanges and the web do not apply any significant restraint to each other at the junction. It is also evident that the flanges and the webs experience a considerable amount of stress loss, particularly at load equal to $3 \mathrm{Pc}$, across their cross-section. The stress loss occurs to such an extent that the stresses become tensile at the flanges free edges. 


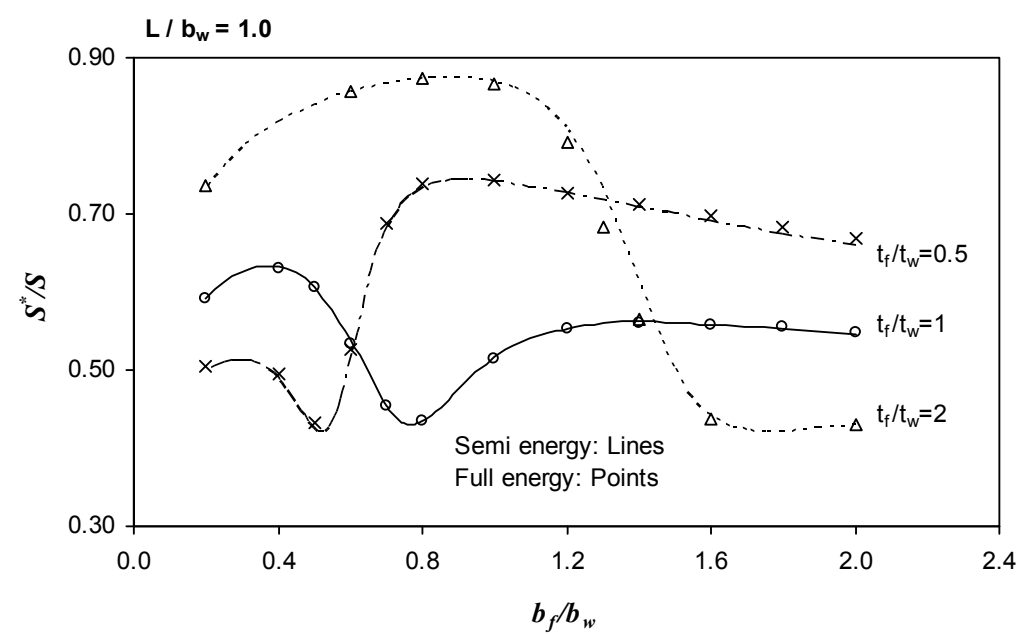

Figure 5. Post-Buckling Compressional Stiffness Ratio of I-Section Struts at the Instant of Buckling

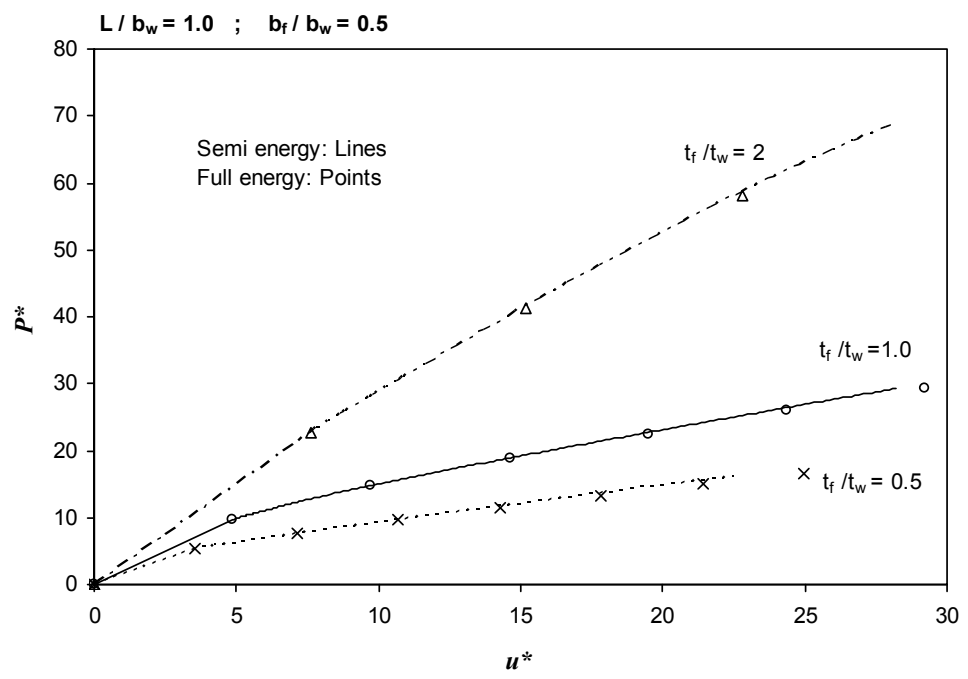

Figure 6. Load-Displacement Variation for I-Section Struts
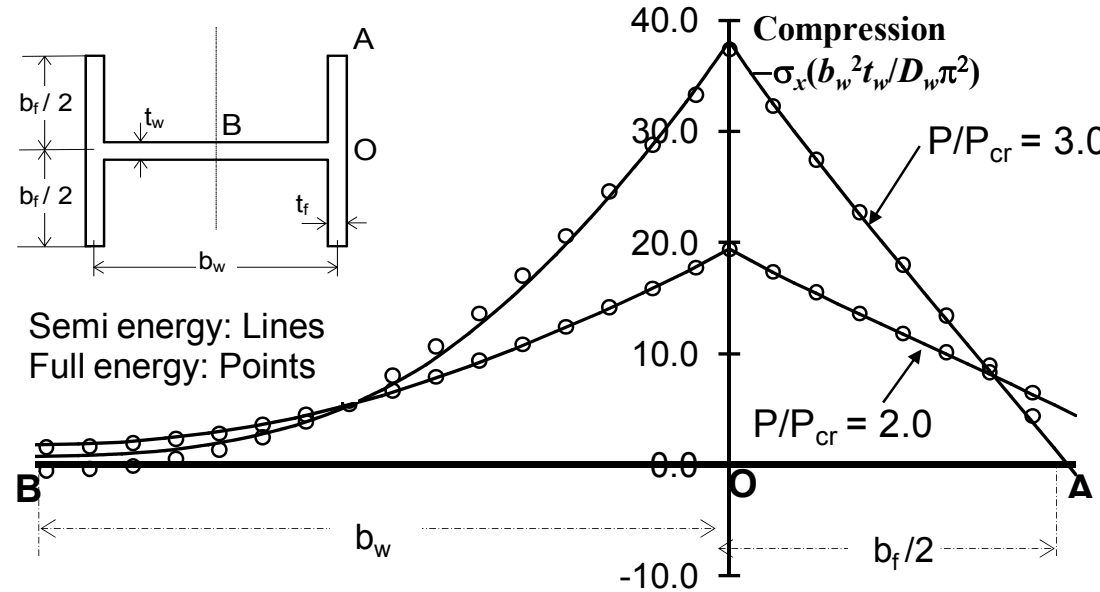

Figure 7. Longitudinal Membrane Stress Distribution at the Crest of the Buckle for I- Section Struts 


\section{CONCLUSIONS}

Two finite strip methods, namely the full-energy and the semi-energy FSM, are developed for predicting the geometrically non-linear response of I-sections with simply supported ends when subjected to uniform end shortening in their plane. Although the formulations of both finite strip methods are based on the concept of the principle of minimum potential energy, the first finite strip method utilizes a semi-energy finite strip, whereas in the second finite strip method (which is designated by the name full-energy finite strip method) all the displacements are postulated by the appropriate shape functions. The developed finite strip methods are then applied to analyze the post-local-buckling behavior of some representative I-sections. Through the comparison of the results, it is observed that for the geometries of the structure under study, the agreement between the results obtained by the semi-energy approach and those obtained by the full-energy method are very good.

However, it is expected that by implementing more than one term in the formulation of the semi-energy approach, and thus by allowing the structure to take up more flexibility if required, the accuracy of the semi-energy approach will improve.

\section{REFERENCES}

[1] Graves Smith, T.R. and Sridharan, S., "A Finite Strip Method for the Post-Locally-Buckled Analysis of Plate Structures", Int. J. Mech. Sci., 1978, Vol. 20, pp. 833-842.

[2] Sridharan, S., "A Finite Strip Analysis of Locally Buckled Plate Structures subject to Nonuniform Compression”, Eng. Struct., 1982, Vol. 4, pp. 249-255.

[3] Graves Smith, T. R. and Sridharan, S., "The Local Collapse of Elastic Thin Walled Columns", J. STRUCT. MECH., 1980, Vol. 8, No. 4, pp. 471-489.

[4] Hancock, G. J., "Nonlinear Analysis of Thin Sections in Compression", Journal of the Structural Division, ASCE, 1981, Vol. 107, No. ST3, pp. 455-471.

[5] Dawe, D. J., and Lam, S. S. E. and Azizian, Z. G., "Finite Strip Post-Local-Buckling Analysis of Composite Prismatic Plate Structures", Computers \& Structures, 1993, Vol. 48, No. 6, pp. 1011-1023.

[6] Kwon, Y. B. and Hancock, G. J., "Post-Buckling Analysis of Thin-Walled Channel Sections Undergoing Local and Distortional Buckling", Research Report, No. R650, School of Civil and Mining Engineering, The University of Sydney, Australia, 1992.

[7] Azhari, M. and Bradford, M. A., "The Use of Bubble Functions for the Post-Local Buckling of Plate Assemblies by the Finite Strip Method”, Int. J. Numer. Meth. Engng., 1995, Vol. 38, pp. 955-968.

[8] Rhodes, J. and Harvey, J. M., "Examination of Plate Post-Buckling Behaviour", J. of Eng. Mech. Div. ASCE, 1977, Vol. 103, No. EM3, pp. 461-478.

[9] Marguerre, K., "The Apparent Width of Plates in Compression", NACA Technical Memorandum No. 833, National Advisory Committee for Aeronautics, Washington, D.C., 1937.

[10] Ovesy, H. R., and Loughlan J., and GhannadPour, S. A. M., "Geometric Non-Linear Analysis of Thin Flat Plates Under End Shortening, Using Different Versions of the Finite Strip Method", International Journal of Mechanical Sciences, 2005, Vol. 47, pp. 1923-1948.

[11] Ovesy, H. R., and Loughlan J., and GhannadPour, S. A. M., "Geometric Non-Linear Analysis of Channel Sections Under End Shortening, Using Different Versions of the Finite Strip Method", Computers \& Structures, 2006, Vol. 84, pp. 855-872. 
[12] Ovesy, H. R., and Loughlan J., and GhannadPour, S. A. M., and Morada, G., "Geometric Non-Linear Analysis of Box Sections Under End Shortening, Using Three Different Versions of the Finite Strip Method", Thin Walled Structures, 2006, Vol. 44, pp. 623-637.

[13] Ovesy, H. R., GhannadPour, S. A. M., "Geometric Nonlinear Analysis of Imperfect Composite Laminated Plates, Under End Shortening and Pressure loading, Using Finite Strip Method", Composite Structures, 2006, Vol. 75, pp. 100-105. 